\title{
Desarrollo profesional: una herramienta efectiva para el crecimiento integral de los profesionales en inglés en la UNED
}

\author{
Tobías Brizuela-Gutiérrez* \\ (iD) https://orcid.org/0000-0001-7291-0634
}

Recibido: 11 de febrero, 2019 - Aceptado: 21 de febrero, 2019

\section{RESUMEN}

El desarrollo profesional es una oportunidad valiosa que invita a los docentes en la enseñanza del inglés a seguir actualizándose en temáticas relacionadas con su campo de acción y les permite además seguir creciendo en su perfil académico y profesional. Dichas oportunidades, sean estos talleres especializados, seminarios de actualización, seminarios en línea, conversatorios o sesiones magistrales; son, sin duda, espacios que abordan una variedad de temáticas que vienen a complementar y enriquecer enormemente la labor docente.

Palabras clave: Desarrollo profesional, seminarios, talleres, crecimiento, actualización.

\section{Formato de citación según APA}

Brizuela Gutiérrez, T. (2019). Desarrollo profesional: una herramienta efectiva para el crecimiento integral de los profesionales en inglés en la UNED. Revista Espiga, 18 (37), 99-110

\section{Formato de citación según Chicago-Deusto}

Brizuela Gutiérrez, Tobías (2019). «Desarrollo profesional: una herramienta efectiva para el crecimiento integral de los profesionales en inglés en la UNED». Revista Espiga 18, n. 37 (enero-junio, 2019): 99-110.

* Bachiller en la Enseñanza del Inglés para I y II ciclos, Universidad Estatal a Distancia (UNED). Licenciatura en Educación con énfasis en Currículo y Evaluación, Universidad de las Ciencias y el Arte. Máster en Educación con énfasis en la Enseñanza del Inglés, Universidad Latina de Costa Rica. Profesor titular del área de Enseñanza del Inglés y encargado de la Cátedra de Enseñanza del Inglés y docente de grado de asignaturas relacionadas con la carrera de Enseñanza del Inglés para I y II ciclos, UNED, Costa Rica. Correo: tbrizuela@uned.ac.cr 
Una frase célebre, de autor desconocido, dice: «You should never stop learning, learning is not just a gift, it's a privilege» («Usted nunca debe parar de aprender, pues aprender no es un regalo, es un privilegio»).

Una actitud de nunca parar de aprender garantiza, sin duda, un profesional actualizado, competente, al día con las nuevas tendencias y siempre en crecimiento.

Una vez concluido el ciclo de estudios universitarios, y dar inicio al viaje llamado «experiencia laboral», el profesional se enfrenta contra una realidad en la que se percibe que todavía hay áreas que necesitan ser repasadas de nuevo, otras enriquecidas e incluso algunas otras aprendidas. Después de invertir cuatro, cinco o seis años en la universidad, preparándose para el campo profesional, es evidente que muchos aspectos quedan pendientes por conocer, explorar e involucrarse.

El desarrollo profesional es, sin duda, la oportunidad para llenar «vacíos», para complementar la preparación universitaria, para ampliar los horizontes de lo que significa el crecimiento integral. El modelo pedagógico de la Universidad Estatal a Distancia (UNED) deja muy clara esta visión de crecimiento y tiene muy claro el valor del desarrollo profesional para el buen desempeño docente. En la sección La construcción del modelo pedagógico en la práctica educativa, apartado 4 , indica que las implicaciones del modelo para llevar a cabo coordinadamente las acciones de diseño de cursos, elaboración de materiales didácticos y la facilitación, seguimiento y atención de los estudiantes, pueden significar funciones, tiempos, remuneración y modalidades de vinculación diferentes. Por la naturaleza de la Institución, la administración debe ser la estructura facilitadora para el desarrollo académico ${ }^{1}$.

Las estructuras de los planes de estudios de los diferentes grados tienen como fin preparar a los futuros profesionales en todos los campos de acción posibles. Los diferentes bloques procuran proveer las herramientas indispensables para desempeñarse efectivamente en la profesión. Se procura que el futuro profesional esté preparado lo mejor posible para enfrentar todas las circunstancias que puedan ocurrir.

Sin embargo, la premisa anterior sería enteramente correcta si se pudieran delinear con anterioridad todas las posibles circunstancias por ocurrir o aparecer en escena y cómo enfrentarlas de la mejor manera. Al considerar tal panorama, el desarrollo profesional representa una excelente y efectiva oportunidad para proveer a los profesionales de herramientas que les permita responder con éxito a las necesidades y oportunidades que se les presenten.

El propósito del presente artículo es ilustrar cómo el desarrollo profesional es una herramienta excelente y sumamente efectiva para el crecimiento integral de los profesionales de la carrera de la Enseñanza del Inglés en la UNED, una vez que se gradúan. Además, se aborda cuáles han sido las iniciativas realizadas y los logros alcanzados.

1. Modelo Pedagógico UNED, pág. 20. 


\section{Desarrollo profesional}

Nicolás Fernández Losa, de la Universidad de la Rioja, define claramente qué es el desarrollo profesional, cuando lo explica como un esfuerzo organizado y formalizado que se centra en el desarrollo de trabajadores más capacitados. Tiene un alcance mayor y de duración más extensa que la formación. El desarrollo debe ser una opción empresarial estratégica si la organización quiere sobrevivir en un entorno global y cada vez más competitivo ${ }^{2}$.

El desarrollo profesional es el proceso por el que las personas progresan a través de una serie de etapas caracterizadas por distintas tareas de desarrollo, actividades y relaciones.

Linda Castañeda y Jordi Adell plantean que el desarrollo profesional docente es un concepto amplio y complejo que engloba múltiples significados, procesos y perspectivas $^{3}$. Se puede definir como el intento de analizar y comprender cómo los docentes, en el ejercicio de su profesión, siguen aprendiendo, aprenden a aprender y transforman sus conocimientos en mejoras en sus prácticas con el objetivo de optimizar los resultados de aprendizaje de los estudiantes. Es decir, un proceso de aprendizaje de los docentes, cuyo objetivo es el cambio en su actuación profesional (mejora del proceso de enseñanza y aprendizaje), que se enmarca en su formación continua (en esa indefinición temporal que representa el aprendizaje a lo largo de toda su vida laboral $\left.{ }^{4}\right)$.

Muy valiosas son también las ideas y aportes de Xie Qian y Zhou Li en su libro Modelo de formación y desarrollo a implementar en las universidades ${ }^{5}$.

La capacitación es una herramienta imprescindible de cambio positivo en las organizaciones. Hoy, no puede concebirse solamente como entrenamiento o instrucción, supera a estos y se acerca e identifica con el concepto de educación.

La tarea de la función de capacitación es mejorar el presente y ayudar a construir un futuro en el que la fuerza de trabajo esté formada y preparada para superarse continuamente. Esta debe desarrollarse como un proceso, siempre en relación con el puesto y con los planes de la organización. En la actualidad, la capacitación es la respuesta a la necesidad que tienen las empresas o instituciones de contar con un personal calificado y productivo, es el desarrollo de tareas con el fin de mejorar el rendimiento productivo, al elevar la capacidad de los trabajadores mediante la mejora de las habilidades, actitudes y conocimientos ${ }^{6}$.

2. Nicolás Fernández Losa, «El desarrollo profesional de los trabajadores como ventaja competitiva de las empresas», Cuadernos de Gestión (2002), acceso: 10 mayo, 2019, http://www.ehu.eus/cuadernosdegestion/documentos/214.pdf

3. Castañeda, Linda y Jordi Adell, «El desarrollo profesional de los docentes en entornos personales de aprendizaje (PLE)», 2011, acceso: 10 mayo, 2019, https://www.researchgate.net/publication/224813636_El_desarrollo_profesional_de_los_docentes_en_entornos_personales_de_aprendizaje_PLE

4. Ibíd.

5. Xie Qian y Zhou Li, Modelo de Formación y Desarrollo a implementar en las universidades (2008), acceso: 10 de mayo, 2019, http://www.eumed.net/libros-gratis/2008b/406/index.htm

6. Ibíd, 15 . 
En el campo de la formación de profesores, varios términos se han usado como sinónimos de desarrollo profesional del docente. Por una parte, se tiene las definiciones próximas al concepto de formación (reciclaje de profesores, perfeccionamiento del profesorado, formación permanente, formación continua, formación en servicio); y, de otro, aquellas acepciones que integran el término desarrollo (desarrollo de profesores, desarrollo profesional-personal, desarrollo del staff, crecimiento profesional $^{7}$ ).

\section{Desarrollo profesional en Costa Rica}

En Costa Rica son muchas las instituciones involucradas en procesos diferentes de capacitación, preparación y profesionalización. Todas cuentan con programas, planes, iniciativas de mejoramiento y desarrollo profesional.

Por ejemplo, el Colegio de Ciencias Económicas de Costa Rica tiene como fin proveer de espacios de intercambio, actualización y especialización profesional en las distintas áreas de las ciencias económicas. La misión del Departamento de Desarrollo Profesional es ofrecer más y mejores oportunidades de actualización e intercambio en las distintas disciplinas que componen las ciencias económicas. El trabajo se sustenta en las iniciativas y demandas presentadas por las personas colegiadas, estructuradas en planificaciones mensuales y semestrales ${ }^{8}$.

Por su parte, el Colegio de Licenciados y Profesores en Letras, Filosofía, Ciencias y Artes (Colypro), promueve e impulsa el desarrollo profesional de las personas colegiadas a través de cursos y actividades de índole académica para lograr un mejor aprovechamiento profesional e individual. Para ello, el Departamento de Desarrollo Profesional y Humano gestiona diversas actividades que fortalecen la formación académica de las personas colegiadas; entre ellas: foros, encuentros, conferencias y videoconferencias, jornadas académicas, cursos virtuales y talleres, entre otras.

Destacan las sesiones de la Cátedra Libre «Victoria Garrón Orozco» sobre la calidad de la educación en las diferentes regiones del país. En tal cátedra, la coordinadora Mayra Montiel Oviedo, indica que se brinda apoyo académico-profesional para actividades de educación continua y capacitación de corta y larga duración en temas relacionados con pedagogía, legislación, administración, relaciones humanas, evaluación, temas específicos u otros que sean de interés para el mejoramiento de la educación y la cultura9 ${ }^{9}$.

El Ministerio de Educación es una institución que alberga más de 50000 docentes en su planilla. Patricia Badilla Gómez, directora ejecutiva del Instituto de Desarrollo Profesional (IDP), en el Informe de detección de necesidades de capacitación 2015, plantea que el desarrollo profesional se entiende como un proceso continuo que implica la

7. Raúl Eirín Nemiña, Herminia García Ruso y Lourdes Montero Mesa, «Desarrollo profesional y profesionalización docente. Perspectivas y problemas», Profesorado. Revista de Currículum y Formación de Profesorado, vol. 13, núm. 2, (2009): 113, acceso: 10 de mayo, 2019, http://www.redalyc.org/pdf/567/56711798016.pdf

8. Sitio web Colegio de Ciencias Económicas, acceso: 10 de mayo, 2019, https://www.colegiocienciaseconomicas.cr/beneficios/desarrollo-profesional/

9. Sitio web Colegio de Licenciados y Profesores en Letras, Filosofía, Ciencias y Artes, acceso: 10 de mayo, 2019, http://colypro.com/servicios/desarrollo-profesional-y-humano 
reflexión introspectiva y participativa del sector profesional de la educación sobre su propia práctica, así como el análisis de las propias creencias, pensamientos, conocimientos, la cultura de la institución y la comunidad educativa. Por consiguiente, es un proceso sistemático, planificado y organizado, cuyo propósito es la actualización y el mejoramiento de prácticas ${ }^{10}$.

En dicho informe, la siguiente figura muestra las prioridades para el personal docente por etapa de desarrollo profesional, en relación con el saber conocer.

\section{Etapa 1}

1. Conocer los últimos avances relacionados con teorías, técnicas, estrategias y recursos de la especialidad.

2. Conocer estrategias de investigación, sistematización y evaluación para mejorar la práctica pedagógica.

3. Conocer estrategias innovadoras de gestión para mejorar los centros educativos.

\section{Etapa 2}

1. Conocer los últimos avances relacionados con teorías, técnicas, estrategias y recursos de la especialidad.

2. Conocer estrategias de investigación, sistematización y evaluación para mejorar la práctica pedagógica.

3. Conocer estrategias innovadoras de gestión para mejorar los centros educativos.

\section{Etapa 3}

1. Conocer los últimos avances relacionados con teorias, técnicas, estrategias y recursos de la especialidad.

2. Conocer estrategias de investigación, sistematización y evaluación para mejorar la práctica pedagógica.

3. Conocer estrategias innovadoras de gestión para mejorar los centros educativos.

\section{Etapa 4}

1. Conocer los últimos avances relacionados con teorías, técnicas, estrategias y recursos de la especialidad.

2. Conocer estrategias de investigación, sistematización y evaluación para mejorar la práctica pedagógica.

3. Conocer estrategias innovadoras de gestión para mejorar los centros.

Figura 1. Fuente: Informe de detección de necesidades de capacitación 2015, figura 6, p. 30.

En el informe Estado de la Nación 2016, Vera Brenes hace referencia al objetivo del Plan Nacional de Formación Permanente 2016-2018, denominado «Actualizándonos», del Instituto de Desarrollo Profesional Uladislao Gámez Solano, del Ministerio de Educación Pública, e indica:

Diferentes investigaciones internacionales coinciden y reiteran que la calidad de la educación de un país está asociada directamente con la calidad de sus profesores, dado que es mediante

10. Patricia Badilla Gómez, «Informe de Detección de necesidades de capacitación», MEP, IDP (2014), acceso: 10 de mayo, 2019, http://idp.mep.go.cr/sites/all/files/idp_mep_go_cr/publicaciones/informe_dnc_2014.pdf 
la práctica docente que se puede o no lograr un buen desempeño de los estudiantes (Velaz y Vaillant, 2009; Mackinsey, 2007; Unesco, 2012; Bruns y Luque, 2014). En Costa Rica, el Plan Nacional de Desarrollo 2015-2018 «Alberto Cañas Escalante» plantea que, para alcanzar una educación de calidad, es necesario promover un desarrollo profesional docente continuo y pertinente, y posiciona el tema como prioritario en las políticas públicas para el período gubernamental 2014-2018 .

A continuación, la siguiente figura (Fig. 2) ilustra las principales temáticas abordadas en tal iniciativa.

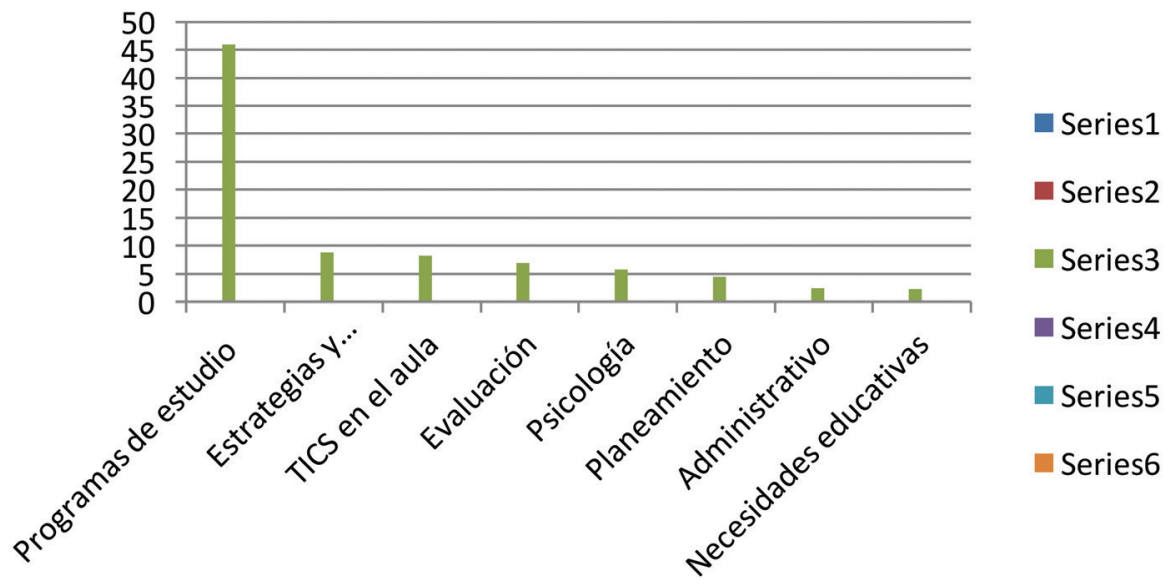

Figura 2. Actividades formales de desarrollo profesional por temática, 2016 (porcentajes).

Fuente: Estado de la Nación, cuadro 7.17.

\section{El desarrollo profesional en la Universidad Estatal a Distancia (UNED)}

\section{Descripción del programa}

La Escuela de Educación, a través del Programa de Capacitación y Desarrollo Profesional $1^{12}$, tiene como objetivo conformar una sociedad de aprendizaje desde la cual se articulen propuestas académicas generadas a partir de la experiencia y conocimientos colectivos compartidos.

Desde esta perspectiva, y enmarcada en la transformación educativa, resulta necesario el enriquecimiento constante de las prácticas pedagógicas. Es por esta razón que «se propone una nueva manera de pensar la formación continua para responder a las necesidades del profesorado a sus contextos de actuación al concebirse como una actividad permanente y articulada con la práctica concreta de los docentes» ${ }^{13}$.

11. Plan Nacional de Formación Permanente 2016-2018, acceso: 10 de mayo, 2019, http://idp.mep.go.cr/sites/all/ files/idp_mep_go_cr/publicaciones/actualizandonos_version_final_3.pdf

12. Sitio web Escuela de Educación, acceso: 10 de mayo, 2019, https://www.uned.ac.cr/ece/desarrollo-profesional

13. Lombardi y Abrile, 2009, acceso 10 de mayo 2019, https://www.uned.ac.cr/ece/desarrollo-profesional 
La carrera de Enseñanza del Inglés tiene el sello de acreditación por parte del Sistema Nacional de Acreditación de la Educación Superior (SINAES), lo que exige procesos de calidad académica para sus estudiantes y graduados. En algunos de los apartados de los informes de acreditación, hay aspectos puntuales en los que se espera de la carrera oportunidades de proyección y de desarrollo profesional para sus graduados. Uno de los objetivos generales de la carrera puntualiza lo siguiente: «Formar educadores y educadoras en la enseñanza del inglés para primaria dispuestos a construir una visión y una actitud pedagógica que los impulse a involucrarse en procesos de educación permanente, que garanticen su desarrollo profesional en forma idónea» ${ }^{14}$.

Una de las primeras iniciativas realizadas en el 2015 desde la carrera de Enseñanza del Inglés, específicamente por la Cátedra de Enseñanza del Inglés en relación con el desarrollo profesional, fue un proyecto de extensión denominado Reflecting on my Daily Work. La iniciativa se propuso instaurar un espacio de reflexión en donde los docentes identificaran sus fortalezas y las oportunidades de mejora dentro de su labor en las aulas. Para esto, se unieron esfuerzos con el Ministerio de Educación Pública (MEP) para desarrollar un ciclo de talleres que, durante un año, estuvo dirigido a profesionales de los circuitos 2 y 3 de la Dirección Regional San José Norte ${ }^{15}$.

La iniciativa buscó que docentes de inglés, de primero y segundo ciclo de la Enseñanza General Básica de la región mencionada, elaboraran, desde sus experiencias, acciones de mejoras que pudieran implementar de manera continua en su quehacer docente. Los talleres de desarrollo profesional abordaron tres temáticas específicas: planeamiento didáctico, diseño de materiales y evaluación de los aprendizajes. Se utilizaron módulos de aprendizaje, espacios de autorreflexión, trabajo colaborativo y actividades en línea complementarias. La población alcanzada fue de 15 docentes, quienes cada mes se reunieron por espacio de seis horas para discutir las temáticas y crecer juntos.

\section{Primer seminario de profesionales}

Con el fin de impactar más población docente en relación con el desarrollo profesional, la carrera, en conjunto con las cátedras de Lengua Inglesa, Lengua y Cultura Inglesa, Enseñanza del Inglés e Inglés Técnico, en el 2017 hizo su primer seminario de desarrollo profesional. Este tuvo lugar en el Paraninfo Daniel Oduber y contó con la participación de 50 personas entre graduados, profesores tutores y estudiantes avanzados de la carrera de Enseñanza del Inglés de la UNED.

A partir de la encuesta de realimentación enviada a los participantes, se obtuvo 28 respuestas. Una de las preguntas planteadas fue: ¿Cuál es su nivel de satisfacción en relación con la relevancia de los temas propuestos y desarrollados en el seminario?

\footnotetext{
14. Informe de reacreditación de la carrera, 2018, pág. 13.

15. «UNED promueve desarrollo profesional en docentes de inglés», Acontecer, acceso: 10 de mayo, 2019, https://www.uned.ac.cr/acontecer/a-diario/juncos/51-a-diario-/ gestion-universitaria/2254-uned-promueve-desarrollo-profesional-en-docentes-de-ingles
} 


\section{CUADRO 1}

Primer Seminario de Profesionales en Inglés, UNED

Tema desarrollado

Necesidades Especiales en la clase de inglés (Special Needs in the English Class)

Habilidades del siglo 21 y evaluación para el aprendizaje del inglés

(The 21st Century Skills and AFL)

Tecnología en la enseñanza del inglés

(Technology in the EFL class)

$\begin{array}{cccc}\begin{array}{c}\text { Bastante } \\ \text { satisfactorio }\end{array} & \begin{array}{c}\text { Muy } \\ \text { satisfactorio }\end{array} & \text { Satisfactorio } & \text { Insatisfactorio } \\ 36 \% & 18 \% & 27 \% & 13 \% \\ 77 \% & 20 \% & 3 \% & - \\ 77 \% & & & - \\ & 23 \% & - & -\end{array}$

Fuente: Datos tomados de las encuestas de satisfacción, Primer Seminario de Profesionales de Inglés (noviembre 2017).

\section{Segundo seminario de profesionales}

En la segunda edición del seminario, la carrera y las cátedras decidieron ampliar su zona de impacto e invitaron, además de graduados, profesores y estudiantes propios de la UNED, a docentes en servicio de otras instituciones privadas. En esta oportunidad se contó con una participación de 70 personas. El evento tuvo lugar en el Paraninfo Daniel Oduber y las temáticas abordadas fueron: evaluación (impartida por el especialista Alfieri Avilán), aprendizaje con tecnologías móviles (impartida por la académica Yorleni Barquero) e integración de las competencias lingüísticas, desarrollada por Josué Sánchez y Octavio Vargas, quienes son graduados de la carrera de Enseñanza del Inglés.

En relación con la realimentación del evento, los participantes expresaron sus opiniones y percepciones de las tres temáticas en función de cinco aspectos puntuales. Seguidamente se muestran sus apreciaciones, cuya escala es: $1=$ Deficiente y $5=$ Excelente.

CUADRO 2

Segundo Seminario de Profesionales en Inglés, UNED

\begin{tabular}{lccc}
\multicolumn{1}{c}{ Aspecto } & $\begin{array}{c}\text { Tema: Evaluación en la } \\
\text { enseñanza del inglés }\end{array}$ & $\begin{array}{c}\text { Tema: Aprendizaje por medio } \\
\text { de tecnologías móviles }\end{array}$ & $\begin{array}{c}\text { Tema: Integración de las competen- } \\
\text { cias lingüísticas en la clase de inglés }\end{array}$ \\
Relevancia del tema & 4.85 & 4.75 & 3.94 \\
Aplicaciones prácticas & 4.68 & 4.60 & 3.52 \\
Utilidad de los materiales & 4.75 & 4.57 & 3.52 \\
Manejo del tiempo & 4.85 & 4.35 & 4.15 \\
Calidad de las respuestas & 4.85 & 4.70 & 3.89 \\
\hline
\end{tabular}

Fuente: Cuestionarios Google Survey enviados por los asistentes al seminario (julio 2018).

16. Opiniones de estudiantes provenientes del Google Survey. 
Sin duda, el seminario fue una gran experiencia de desarrollo profesional. Las opiniones de los asistentes lo corroboran ${ }^{16}$ :

«Gracias por proveernos de nuevos aprendizajes para nuestra labor diaria».

«Este tipo de actividades son importantes pues ayudan a mejorar nuestros procesos de enseñanza».

«Verdaderamente disfruté el seminario».

El espacio que propicia el seminario de profesionales se convierte no solo en una oportunidad de crecimiento profesional, sino en una ventana para socializar con otros colegas, quienes trabajan en otras latitudes y bajo otras condiciones; lo cual permite conocer la realidad del contexto nacional en lo que se refiere a la enseñanza y aprendizaje del idioma inglés.

A continuación se muestran otras actividades que la carrera ha realizado durante los últimos tres años como alternativas al desarrollo profesional y académico.

CUADRO 3

Actividades de desarrollo profesional, carrera Enseñanza del Inglés

\begin{tabular}{lll}
\multicolumn{1}{c}{ Actividad } & \multicolumn{1}{c}{ Temática } & \multicolumn{1}{c}{ Fecha, lugar y cantidad de participantes } \\
Taller & Impostación de la voz & Mayo 2017 / mayo 2018, Paraninfo UNED \\
& & 20 participantes \\
Taller & $\begin{array}{l}\text { Uso de materiales auténticos para el mejor } \\
\text { desarrollo de las habilidades lingüísticas del inglés }\end{array}$ & $\begin{array}{l}\text { Noviembre 2018, Paraninfo UNED } \\
\text { 25 participantes }\end{array}$ \\
\hline
\end{tabular}

Fuente: Elaboración propia.

Para el 2019 se proyecta trabajar en varias propuestas y temáticas. El cuadro 4 visualiza dicha proyección.

CUADRO 4

Actividades de desarrollo profesional 2019, carrera Enseñanza del Inglés, UNED

\begin{tabular}{|c|c|c|}
\hline Nombre de la actividad & Fecha y hora & Lugar \\
\hline $\begin{array}{l}\text { I Taller: Developing Vocabulary Strategically } \\
\text { A cargo de: Alfieri Avilán }\end{array}$ & $\begin{array}{l}30 \text { de enero, } \\
\text { de } 3: 30 \text { p.m. a 5:00 p.m. }\end{array}$ & Paraninfo, aula 2 \\
\hline $\begin{array}{l}\text { II Taller: The First Glimpse of Neuroscience and Language Learning } \\
\text { A cargo de: Alfieri Avilán }\end{array}$ & $\begin{array}{l}31 \text { de enero, } \\
\text { de } 3: 30 \text { p.m. a 5:00 p.m. }\end{array}$ & Paraninfo, aula 2 \\
\hline $\begin{array}{l}\text { III Taller: Propuesta para el uso del miniprojects dentro } \\
\text { del componente de Trabajo Cotidiano. } \\
\text { A cargo de: Roxy Calderón y Ariel Martínez }\end{array}$ & $\begin{array}{l}9 \text { de marzo, } \\
\text { de 8:00 a.m. a 12:00 p.m. }\end{array}$ & Paraninfo, aula 4 \\
\hline $\begin{array}{l}\text { IV Taller: Taller de gramática: To Be or Not to Be. } \\
\text { A cargo de: Elizabeth Cascante y Andrea Lasso de la Vega }\end{array}$ & $\begin{array}{l}14 \text { de marzo, } \\
\text { de 2:00 p.m. a 5:00 p.m. }\end{array}$ & Ciencias Exactas \\
\hline $\begin{array}{l}\text { V Taller: Impostación de la voz para docentes. } \\
\text { A cargo de: Rose Mary Mayorga }\end{array}$ & $\begin{array}{l}31 \text { de mayo, } \\
\text { de 2:00 p.m. a 5:00 p.m. }\end{array}$ & Ciencias Exactas \\
\hline $\begin{array}{l}\text { VII Taller: Webinar } \\
\text { A cargo de: Paola Serrano y Andrea Lasso de la Vega }\end{array}$ & $\begin{array}{l}18 \text { de octubre, } \\
\text { de 2:00 p.m. a 5:00 p.m. }\end{array}$ & $\begin{array}{l}\text { Paraninfo, aula } 2 \\
\text { (o modalidad Webinar) }\end{array}$ \\
\hline
\end{tabular}

Fuente: Elaboración propia. 


\section{Conclusiones}

A continuación, se puntualizan las conclusiones principales que se desprenden de este artículo.

1. Las actividades que se provean a los graduados de la carrera de Enseñanza del Inglés serán siempre excelentes mecanismos para el crecimiento y desarrollo profesional y académico. Las temáticas que se aborden deben ser variadas, retadoras, prácticas $\mathrm{y}$, sobre todo, con una intención de proveer herramientas que ayuden a mejorar los procesos de enseñanza y aprendizaje. En la medida de lo posible, dichas actividades pueden provenir de un diagnóstico de los mismos participantes sobre las temáticas más urgentes y necesarias en la labor docente.

2. Ofrecer oportunidades de desarrollo profesional debe ser un compromiso de la carrera de la Enseñanza del Inglés y se deben hacer esfuerzos para que se ofrezcan no solamente en la sede central de la UNED, sino que se proyecten a otras regiones, comunidades y centros universitarios. Esto con el fin de alcanzar y beneficiar a profesionales en otras latitudes distantes. Se podrían plantear las actividades en formato de Webinar, ya sea por medio del Departamento de Video Conferencias o haciendo uso de tecnologías y salas de conferencia como las que presenta la plataforma ZOOM. Esta iniciativa respondería más a la visión de la UNED.

3. Las actividades que se programen, sean talleres, ponencias o seminarios, deben responder a necesidades reales de los beneficiados. De modo que también se hace imperante la necesidad de contar con instrumentos de realimentación al final de las actividades, con el fin de determinar el nivel de relevancia de las temáticas, su utilidad en los procesos de enseñanza aprendizaje y posibles temáticas para ser consideradas a futuro.

4. Las redes y vínculos con otras instituciones, dependencias, centros de enseñanza, universidades y casas publicadoras, entre otras, permiten a la carrera, además de fortalecer alianzas estratégicas, compartir esfuerzos y beneficiarse de los recursos que estas pueden proveer en función del desarrollo profesional de los docentes de inglés. Vínculos con la Asociación Costarricense de Profesores de Inglés (ACPI), Centro Cultural Costarricense Norteamericano (CCCN) y Universidad de Costa Rica, así como editoriales educativas, deben fortalecerse con el fin de compartir recursos y oportunidades académicas de crecimiento. 


\section{Professional Development: A Tool for an Effective Improvement of English Teachers at UNED.}

Professional development is an enriching and essential opportunity that invites English teachers to grow and update themselves in the different topics related to their expertise. Moreover, it encourages teachers and professors to foster their academic and professional performance. Workshops, seminars, Webinars, virtual sessions, lectures, PD Talks are among some of the options English teachers can benefit from while complementing and strengthening their teachinglearning journey.

Key words: Professional development, seminars, workshops, growing, updating.

\section{RÉSUMÉ}

\section{Le développement profesionnel: un outil effectif pour la croissance intégrale des professionnels en anglais dans l'UNED}

Le développement professionnel est une opportunité qui invite les enseignants d'anglais à continuer à s'actualiser dans des thématiques rapportées à leur domaine; ceci leur permet de développer leur profil académique et professionnel. Que cela soit à travers d'atéliers specialisés, de séminaires de mise à jour, de sémainaries en ligne, de forums ou d'exposés magistrales, ces opportunités sont, sans doute, des espaces qui abordent un éventail de sujets qui complémentent et enrichissent le travail des enseignants.

Mots-clés: Développement professionnel, sémainaires, atéliers, croissance, mise à jour.

\section{Referencias}

Badilla Gómez, Patricia. «Informe de Detección de necesidades de capacitación», MEP, IDP (2014). Acceso: 10 de mayo, 2019. http://idp.mep.go.cr/sites/all/files/idp_mep_go_cr/publicaciones/informe_dnc_2014.pdf

Castañeda, Linda y Jordi Adell. «El desarrollo profesional de los docentes en entornos personales de aprendizaje (PLE)», 2011. Acceso: 10 mayo, 2019. https://www.researchgate.net/publication/224813636_El_desarrollo_profesional_de_los_docentes_en_entornos_personales_de_aprendizaje_PLE

Estado de la Nación, «La voz de los actores del sistema educativo» (2016). Acceso: 10 de mayo, 2019. https:// www.estadonacion.or.cr/educacion2017/assets/parte-2-capitulo-7.pdf

Nicolás Fernández Losa. «El desarrollo profesional de los trabajadores como ventaja competitiva de las empresas», Cuadernos de Gestión (2002). Acceso: 10 mayo, 2019. http://www.ehu.eus/cuadernosdegestion/ documentos/214.pdf

Programa de Capacitación y Desarrollo Profesional, acceso 10 de mayo 2019, https://www.uned.ac.cr/ece/ desarrollo-profesional

Raúl Eirín Nemiña, Herminia García Ruso y Lourdes Montero Mesa. «Desarrollo profesional y profesionalización docente. Perspectivas y problemas», Profesorado. Revista de Currículum y Formación de Profesorado, vol. 13, núm. 2, (2009): 113. Acceso: 10 de mayo, 2019. http://www.redalyc.org/pdf/567/56711798016. pdf

Sitio web Escuela de Educación. Acceso: 10 de mayo, 2019. https://www.uned.ac.cr/ece/desarrollo-profesional

Sitio web Colegio de Ciencias Económicas. Acceso: 10 de mayo, 2019. https://www.colegiocienciaseconomicas. $\mathrm{cr} /$ beneficios/desarrollo-profesional/ 
Sitio web Colegio de Licenciados y Profesores en Letras, Filosofía, Ciencias y Artes. Acceso: 10 de mayo, 2019. http://colypro.com/servicios/desarrollo-profesional-y-humano

Universidad Estatal a Distancia. Carrera de Enseñanza del Inglés. Informe de Reacreditación de la carrera (2018): 13 .

Universidad Estatal a Distancia. Modelo Pedagógico (febrero 2005): 20.

«UNED promueve desarrollo profesional en docentes de inglés». Acontecer. Acceso: 10 de mayo, 2019. https://www.uned.ac.cr/acontecer/a-diario/juncos/51-a-diario-/ gestion-universitaria/2254-uned-promueve-desarrollo-profesional-en-docentes-de-ingles

Xie Qian y Zhou Li. Modelo de Formación y Desarrollo a implementar en las universidades (2008). Acceso: 10 de mayo, 2019. http://www.eumed.net/libros-gratis/2008b/406/index.htm 